Regular Paper

\title{
The Medial Amygdala Controls Coital Access of Female Rats: A Possible Involvement of Emotional Responsiveness
}

\author{
Yasuhiko Kondo and Yasuo SAKUMA
}

Department of Physiology, Nippon Medical School, Sendagi 1, Bunkyo-ku, Tokyo, 113-8602 Japan

Received on Nov 10, 2005; accepted on Dec 20, 2005; released online on Jan 17, 2006; DOI: 10.2170/jjphysiol.RP001105

Correspondence should be addressed to: Yasuhiko Kondo, Tel: +81-3-3822-2131 (Ext. 5356), Fax: +81-3-5685-3055, E-mail: ykondo@nms.ac.jp

\begin{abstract}
Partner preference and paced mating tests were accomplished in ovariectomized female rats following bilateral radiofrequency lesions in the medial amygdala. Open field behavior and passive avoidance learning were also examined to investigate the underlying behavioral mechanism. Partner preference was determined in a chamber located between castrated and sexually active males. Airborne olfactory cues were presented to the female through small holes on the partition. The lesion diminished preference for the odor of sexually active males over that of castrated males, even after injection with high-dose of estrogen. On the other hand, in a paced mating test, the lesioned females without estrogen treatment showed a significantly shorter latency for entering the male's compartment in a two-compartment apparatus, which allowed the females, but not the males, to cross the barrier through a narrow opening at the bottom. However, administration of estrogen and progesterone reduced the effect. The lesion had no effect on emotionality or exploratory behavior in an open field test, but impaired passive avoidance learning capability. We suggest that a male poses an inherent threat to a female. The seemingly incompatible results of partner preference and paced mating tests can be compromised if the male is inherently aversive to the female; this emotional response can be removed by the medial amygdala lesion. [The Japanese Journal of Physiology 55(6), 2005, in press]
\end{abstract}

Key words: female sexual behavior, partner preference, paced mating, medial amygdala, estrogen.

In the wild rat, sexual interactions are initiated and paced by females in estrus through patterns of approach toward and withdrawal from sexually active males [15]. Although some components of female sexual behavior imply the appetitive aspect, the initiative by females, however, is obscured by male active pursuit when tested in ordinary laboratory situation such as in an aquarium. To solve this problem, several researchers are directing attention to paced mating situation, mimicking wild environment $[14,38]$. In the paced mating, female rats can stay a place inaccessible for males and visit a compartment for mating with stimulus males. The situation allows females to regulate their own copulatory pacing without disturbance by males. Pacing behavior, then, is thought to reflect their sexual motivation.

Olfactory preference toward sexually active males is also affected by female sexual motivation. When presented odors of sexually active and inactive males, sexually motivated females spend longer time to explore odors from sexually active males. This test also measures female sexual motivation without influence by stimulus male behavior [43].

Since the body of our knowledge on neural circuits for female sexual behavior has been accumulated by studying lordosis, a reflexive component of female rat sexual behavior, some researchers start on reevaluation of the regulatory mechanisms of female sexual behavior by means of these test paradigms [16, $21,44,45]$. The lateral septum and the medial preoptic area both have been known to belong to lordosis inhibitory system because destruction of the areas facilitate lordosis in female rats. However, our recent study using paced mating and preference tests revealed that these areas may play different roles in neural regulation of female sexual behavior: lesion in the lateral septum affected pacing behavior but not sexual preference, while lesion in the medial preoptic area eliminated sexual preference but did not influence pacing behavior. 
In the present study, we investigated the role of the medial amygdala in regulating sexual behavior in female rats. The medial amygdala has been known to play a critical role in copulatory behavior in male rats. The region processes pheromonal signals received in the vomeronasal organ and also contains a large amount of steroid receptors, expecting an integral role of chemosensory and humoral information. Thus, the present experiment examined effects of bilateral lesions in the medial amygdala on pacing behavior and sexual preference, an appetitive component of female sexual behavior in the rat.

\section{METHODS}

Animals and brain surgery. Male and female Wistar rats ( 9 weeks old at the start) were obtained from the Institute for Animal Reproduction (Ibaraki, Japan) and maintained under controlled temperature $\left(23-24^{\circ} \mathrm{C}\right)$ and reversed illumination (lights on, 23:00-11:00). Food and water were available ad libitum. Stimulus stud males were screened for their vigorous mating activity. Some of them were orchidectomized under ether anesthesia, at least 3 weeks prior to use as stimulus animals in the preference test. Animal housing and the experiment adhered to the Guidelines for Care and Use of Laboratory Animals of the Institute of Laboratory Animal Resources, National Research Council (1996), and the experimental procedure has been approved by our institutional committee for experimental animal ethic.

All females were ovariectomized and subjected to brain surgery under anesthesia with a combination of ketamine hydrochloride and pentobarbital sodium $(25 \mathrm{mg} / \mathrm{kg}$, each). For the brain surgery, they were secured in a stereotaxic frame with an incisor bar placed $3.3 \mathrm{~mm}$ below the interaural line. The parietal bone was drilled and a lesion electrode $(0.7 \mathrm{~mm}$ in diameter) was inserted into the medial nucleus of the amygdala (MeA), sequentially to the right and left hemispheres. Stereotaxic coordinates were: $2.5 \mathrm{~mm}$ posterior to the bregma, $3.2 \mathrm{~mm}$ lateral to the midline and $9.0 \mathrm{~mm}$ below the surface of the skull. Bilateral radiofrequency lesions were produced by heating the tip to $52^{\circ} \mathrm{C}$ for $1 \mathrm{~min}$ by means of a Radionics lesion generator (Model RFG-4A). Sham-operated animals received the same procedure without heating.

Experimental design for behavioral tests and hormonal regimen. Two weeks after the surgery, paced mating and partner preference tests were begun. A series of behavioral tests were carried out with or without hormonal treatment every other week in the following order: (1) paced mating test without hormonal treatment, (2) partner preference test, $48 \mathrm{~h}$ after S.C. injection of $5 \mu \mathrm{g}$ estradiol benzoate (EB, in $0.1 \mathrm{ml}$ sesame oil), (3) paced mating test, $48 \mathrm{~h}$ after $5 \mu \mathrm{g}$ EB, (4) paced mating test, $48 \mathrm{~h}$ after $20 \mu \mathrm{g} \mathrm{EB}$, (5) partner preference test, $48 \mathrm{~h}$ after $20 \mu \mathrm{g}$ EB supplemented with $500 \mu \mathrm{g}$ progesterone ( $\mathrm{P}$, in $0.1 \mathrm{ml}$ sesame oil) $4 \mathrm{~h}$ prior to the test, and (6) paced mating test after $20 \mu \mathrm{g}$ EB and $500 \mu \mathrm{g}$ P injections. No experimental female encountered the same male more than once as stimulus males in behavioral tests.

Open field test and passive avoidance training (acquisition phase) were carried out in all females, which spent 3 weeks undisturbed after the end of the 6 th test. On the next day, they were tested for the retention of passive avoidance learning (retention phase).

To avoid any distraction, all behavioral tests were carried under remote surveillance by video cameras. Video tape recordings were analyzed off-line by a PC running an event-recorder program.

Partner preference test. The acrylic observation box used for the test was basically similar to the one designed by Sachs [40]. The box, $110 \mathrm{~cm}$ long (L), $12 \mathrm{~cm}$ wide (W), and $30 \mathrm{~cm}$ high (H), was divided into 3 compartments of equal width by 2 opaque partitions. Three panels, with a hole $3 \mathrm{~cm}$ in diameter, bored at the center but at different heights from the floor, were assembled into a partition, which allowed the passage of air and sound but prevented visual and physical interactions. A 2-cm-deep, hollow transparent cylinder was attached to each hole located in the panels facing the middle compartment at height of $2 \mathrm{~cm}$. The side compartments had air-inlets (mesh-covered holes, $10 \mathrm{~cm}$ in diameter) at the ends, and the center compartment had an air-outlet at the top. A motor-driven blower, attached to the air-outlet via a flexible duct, introduced air from the compartments on both sides into the center compartment (approximately $0.2 \mathrm{~m}^{3} / \mathrm{min}$ ) through the hollow cylinders. Partner preference was determined by time spent by experimental females poking their nose into the cylinders.

In each test, an experimental female was put in the middle compartment, and left to acclimate for $5 \mathrm{~min}$. The test was started by introducing a sexually active male or an orchidectomized male into each of the side 
This version is to be replaced by the final version after page-setting and proofing.

compartments. The positions of stimulus males were counterbalanced among the tests. Each test lasted for 5 min. After each test, the apparatus was cleaned and sprayed with $70 \%$ ethanol. Fresh bed shavings were put into the compartments for the next test.

Paced Mating Test. An acrylic observation box, $70 \mathrm{~cm}(\mathrm{~L}) \times 30 \mathrm{~cm}(\mathrm{~W}) \times 30 \mathrm{~cm}(\mathrm{H})$, had a barrier with a guillotine door, which divided the box into 2 compartments, $30 \mathrm{~cm}$ and $40 \mathrm{~cm}$ in long axis. The door could be lifted to make a $2.5-3.0 \mathrm{~cm}$ aperture at the bottom of the barrier, which allowed females, but not males, to cross the barrier.

An experimental female was placed into the $30 \mathrm{~cm}$ compartment and a sexually vigorous male into the 40 cm compartment. The paced mating test was started by opening the door after a 5 min acclimation and lasted for $10 \mathrm{~min}$. Video-tape recordings of female rat behavior were analyzed off-line to determine pacing and female sexual behavior. On pacing behavior, parameters were latency to enter the male compartment, percent exits from the male compartment, which followed different degrees of sexual interactions with the male (i.e. mount, intromission and ejaculation), and return latency to re-entry.

Because intensive sexual contacts by males cause females to exit more often after a shorter stay in the male compartment [47], scores of sexual behavior, which included both proceptive and receptive components were observed. The evaluation of proceptivity depended on the time spent for solicitatory locomotion, i.e. darting and hopping, and ear wiggling during the 10 min observation period. The lordosis quotient (LQ), percent lordosis per 10 mounts, was used to determine receptivity.

Open field test. Open field behavior and passive avoidance learning were tested 3 weeks after the end of tests for sexual behavior, in the absence of circulating sex hormones [41]. All females were tested in a circular open field made of white polyvinyl chloride boards, $90 \mathrm{~cm}$ in diameter and surrounded by a $50 \mathrm{~cm}$ (H) wall. The floor was marked by 8 radial lines and a $50-\mathrm{cm}$ circle, which divided the arena into the center and rim. At the beginning of the test, each female was placed in the center of the arena. The number of line crossings within the center and rim, rearings and defecation were recorded over a 3 min period.

Passive Avoidance Test. An acrylic box, $50 \mathrm{~cm}(\mathrm{~L}) \times 30 \mathrm{~cm}(\mathrm{~W}) \times 30 \mathrm{~cm}(\mathrm{H})$, had light and dark compartments of similar size, with walls painted either white or black. The two compartments were divided by a guillotine door. A light bulb brightly illuminated the white compartment (approximately 2,500 lx), and the black compartment had a stainless grid floor connected to a scramble grid-floor shocker (Model E13-08, Coulbourn Instruments, PA, USA). An experimental female was placed in the white compartment. After 5-min acclimation, the door was opened and latency to enter the dark compartment was determined. After the female entered the black compartment, the door was closed and 2 brief foot shocks were delivered (1.0 mA, $1 \mathrm{~s}, 10 \mathrm{~s}$ apart). The female remained in the black compartment for another $1 \mathrm{~min}$. After spending a night in their home cage, the subjects were placed again in the white compartment, and latency to enter the black compartment was determined to evaluate retention.

Histological Examination. On completion of all behavioral tests, the animals were deeply anesthetized with an overdose of sodium pentobarbital and perfused transcardially with saline followed by $10 \%$ formalin. The brain was post-fixed in a solution of $30 \%$ sucrose and $10 \%$ formalin, and $50-\mu \mathrm{m}$ thick frozen serial sections were made in the frontal plane. The sections were stained with Cresyl violet, and the position and extent of the MeA lesions and associated damage were determined under microscopic observation.

Statistical Analysis. Lordosis quotients, time spent for soliciting behavior, latency to enter the male compartment and time spent in the male compartment were analyzed by two-way ANOVA with a repeated measure ( 4 hormonal treatments $\times 2$ groups). Time in seconds and $\%$ time for nose-poking toward sexually active males were also analyzed by two-way ANOVA with a repeated measure ( 2 hormonal treatments $\times 2$ groups). Paired t-test was used to compare time for nose-pokings between sexual and castrated males. Return latencies and \% exit after sexual interactions in paced mating test, and parameters in open field test and passive avoidance test were examined by $t$-test. Parameters in open field test were examined by paired t-test, and latency in passive avoidance test was examined by U-test because we put maximal values (300 s) for animals that showed no entrance to the shocked compartment. Finally, we calculated Kendall (rank order) correlation coefficients to examine relationship between passive avoidance score and latency in paced mating test. 


\section{RESULTS}

\section{Partner preference}

The MeA lesion diminished the preference of estrous female rats for sexually vigorous males over orchidectomized males (Fig. 1). Sham-operated females spent a larger percentage of time poking their nose toward or sniffing sexually vigorous males than orchidectomized males. When treated with a low dose $(5 \mu \mathrm{g})$ of EB, the sham-operated females spent $86.5 \%$ of all the nose-poking time with sexually vigorous males; treatment with a combination of a large dose of EB $(20 \mu \mathrm{g})$ and P had a similar effect $(75.9 \%)$. Both values were significantly larger than those toward castrated males $(t=7.01$ and 4.14 , respectively, $d f=5, p<.01)$. The lesion diminished the preference: the values were $58.3 \%$ in the low dose condition $(t<1, d f=5,1$ lesioned female showed no nose-poking, NS) and $58.0 \%$ in the high dose condition $(\mathrm{t}=1.61, d f=6$, NS). The difference between lesioned and sham-operated females was significant by ANOVA $[F(1,11)=8.42, p<$ $0.05]$.

The actual time spent nose-poking toward or sniffing sexually vigorous males was not significantly different for the lesioned and sham-operated female rats $[F(1,11)<1]$. Pretreatment with different hormone regimens had no effect. In the low dose condition, the lesioned females spent $19.6 \pm 7.3 \mathrm{~s}$ nose-poking toward sexually vigorous males, whereas sham-operated females spent $44.0 \pm 9.1 \mathrm{~s}(t=2.12, d f=11, p=$ $0.058, \mathrm{NS})$. The combination of a higher dose of EB and P did not induce preference toward sexually vigorous males (59.7 $\pm 11.4 \mathrm{~s}$ in the lesioned females vs. $60.4 \pm 14.7 \mathrm{~s}$ in the sham-operated females, NS).

Regardless of the pretreatment with different hormone regimens, total time spent nose poking for either sexually vigorous or orchidectomized males was not significantly different between sham-operated and lesioned females.

\section{Sexual and pacing behavior}

The MeA lesion had no effect on either receptivity or proceptivity, throughout various hormonal treatment regimens (Fig. 2). It was not surprising that a combination of a large dose of EB (20 $\mu \mathrm{g})$ and P made the sham-operated females fully receptive with an increase in their proceptivity. The MeA lesion did not cause any changes in the effects of the hormonal treatment.

The paced mating test revealed major effects of MeA lesion both in terms of the latency to enter $[F(1,11)$ $=10.7, p<.01]$ and the time spent in the male compartment $[\mathrm{F}(1,11)=14.2, p<0.01]$ ( Fig. 3). In the sham-operated females, the latency to enter the male compartment depended on the dose of EB, with a threshold between 5 and $20 \mu \mathrm{g}$. Prior EB treatment was not necessary to induce this behavior in the lesioned females, so that the lesioned females entered the male compartment at significantly shorter latencies in the absence of $\mathrm{EB}[F(1,11)=4.4, p<0.05]$ or after treatments with $5 \mu \mathrm{g} \operatorname{EB}[F(1,11)=5.7, p<0.05]$. The reduced latency in the sham-operated females following treatment with a combination of EB and P rendered the difference between the sham-operated and lesioned females insignificant.

The time spent in the male compartment also depended on prior hormonal treatment in the sham-operated females but not in the lesioned females. The lesioned females stayed in the male compartment for a substantially longer time. Statistical difference between the sham-operated and lesioned females were detected as the main effect of lesion in ANOVA $[F(1,11)=14.2, p<0.01]$. The combination of EB and $\mathrm{P}$ increased the rate of exit in the sham females, thus causing a reduced time spent in the male compartment (see below).

Sexual contacts with males, when arranged in the rank of their intensity as mounts, intromissions and ejaculations, caused sham operated females to exit the male compartment more often in that order, in terms of percent exit following these contacts (Fig. 4, upper). The percent exit following intromissions was significantly smaller in the lesioned females than in the sham-operated females $(t=3.27, d f=10, \mathrm{p}<0.01)$. The difference contributed to a longer stay by lesioned females in the male's compartment. The return latency, the time spent by the females in their compartment after exiting the male compartment until re-entry, was not significantly different following different patterns of sexual contact by males (Fig. 4, lower).

\section{Open field behavior and passive avoidance learning}

Parameters of open field behavior were all similar for the sham-operated and lesioned females (Table 1). 
This version is to be replaced by the final version after page-setting and proofing.

The lesioned females had deficits in passive avoidance learning, with a significant shorter latency to enter the dark room than the sham females in the retention phase (Fig. 5).

In order to examine the relationship between passive avoidance performance and entering male compartment in paced mating test, we calculate correlation coefficients of latencies in avoidance and paced mating. Significant correlations were obtained in those in avoidance test vs. paced mating tests without EB treatment $(r=.493, p<0.05)$ and with $5 \mu \mathrm{g}$ EB treatment $(r=.466, p<0.05)$.

\section{Location and extent of the lesions}

Histological examination showed that 7 females (out of 10 operated on) had bilateral radiofrequency lesions restricted to the MeA (Fig. 6). The lesion measured approximately $0.6-1.0 \mathrm{~mm}$ on the major axis and was centered in the posterodorsal part of the MeA. The lesion spared the ventral part of the MeA as well as the molecular layer in which the fibers from the accessory olfactory bulb terminate [22]. Other nuclei of the amygdala were left intact. Electrode penetration produced small scars in the optic tract and internal capsule, which were common to the experimental as well as sham-operated females $(n=6)$. Data from the animals, in which histological examination revealed failure in the placement of bilateral lesion in the MeA, were excluded from further analysis. Thus, final numbers of experimental females were 7 in the lesioned group and 6 in the sham-operated group in following data analyses.

\section{DISCUSSION}

In the present study, MeA lesions diminished the preference of estrous female rats for sexually vigorous males over orchidectomized males, and facilitated the female's entrance into the male compartment independent of pretreatment with sex hormones. The lesioned female stayed in the vicinity of the male for longer time, after sexual interactions. The lesion did not alter exploratory behavior or affect emotionality measured in the open field test, but disrupted passive avoidance performance.

The results showed that the MeA is involved in the estrogen-dependent regulation of partner preference [43]. The significance of estrogen in producing preference for the male has been shown by a runway-choice study, which also detects the level of motivation to seek out males, presumably using odor as a clue [13]. Either local MeA neurons that express estrogen receptor $\alpha$ or $\beta[19,37]$ or estrogen-sensitive MeA afferents with origins in the MPOA [46] or other hypothalamic structures [1] would provide a neural basis for the estrogen action on partner preference. Exposure to male odors increased the number of cells co-expressing Fos and estrogen receptor $\alpha$ in the female rat MeA [2]. In any case, regulation of the partner preference by estrogen would not be straightforward, because estrogen exerts different effects on aversive and appetitive components in sexual interactions as exemplified by prior sexual experience in the induction of a male-directed orientation in the female rat [42].

Aversion and appetite are two determinants of partner preference. The discrepancy between our preference test results and the results by [6], in which estrous female rats spend more time with orchidectomized males than with intact males, could be explained by the difference in the test situation. [6] allowed female rats to sexually interact with tethered males, whereas our preference test depended solely on remote cues, olfactory and auditory. In our paced mating test, sham operated females spent less time in the male compartment depending on the intensity of sexual stimuli they received. Because intact males become by far the partners preferred by females in estrus when intromissions are prevented by vaginal occlusion, excessive genital stimulation has been regarded as aversive to females [6]. Similarly, intromission during copulation correlates negatively with subsequent appetitive sexual behavior [9, 15]. Instrumental responding analysis has shown that females that received mounts with intromission are slower to respond subsequently to a male than females that received mounts without intromission [3]. Although these data are equally consistent with the interpretation that sensory information relevant to intromission could have a consummatory value for the sexual appetite of the females [24, 38], the MeA does not regulate partner preference through modification of sexual motivation, because the lesioned females spent as much time nose poking as the sham operated females. The peculiar role of the MeA in the regulation of partner preference is clear when compared with the medial preoptic area. Lesion of the latter impaired sexual preference and nose poking altogether, suggesting that a lack of sexual preference caused by the medial preoptic lesion is secondary to the loss of sexual motivation [44].

The lesion in the present study centered in the posterodorsal part of the MeA, which has been implicated 
This version is to be replaced by the final version after page-setting and proofing.

not only in the detection of sexually relevant pheromonal cues as the vomeronasal amygdala but also in the integration of a wide range of social behavior [17,35]. A similar lesion in the male rat eliminates non-contact penile erection provoked by odors of estrous females [27], but the normal preference for estrous over anestrous females is maintained [26], thus suggesting impaired processing of estrous odors but not disruption of the central pheromonal pathway. In male rats exposed to inaccessible estrous females, significant Fos expression was found in the posterodorsal, but not in the posteroventral part of the MeA [25]. The volume of the posterodorsal $\mathrm{MeA}$ is greater in male rats than in females, which depend on the circulating titer of androgen [8]. Therefore, sexual difference in the central olfactory pathway cannot be ruled out as a caveat associated with the sex specific loss of preference in females following MeA lesion. All the same, the present results are unlikely to be due to the loss of olfaction.

Emotional coping with fear and anxiety has traditionally been allocated to the central and basolateral amygdala (see [10] for review). The central nucleus is the major output structure of the amygdala with axons to hypothalamic and midbrain nuclei that are critically involved in autonomic, neuroendocrine and behavioral responses evoked by unconditioned or conditioned fear-arousing stimuli [29]. Electrical stimulation of the central amygdala induced, whereas lesions diminished, fear-like responses [23, 30, 36]. The basolateral nucleus is considered to establish association between conditioned and aversive unconditioned stimuli [28, 32]. Lesions [31, 33] or disruption of NMDA transmission [34] in the basolateral nucleus lessened fear-related behavior, such as freezing, when returned to an environment in which they previously received a footshock.

The odor of a predator elicits a battery of defensive behavior which encompasses fear, anxiety, risk assessment, autonomic and neuroendocrine responses [5, 12]. Sex difference in the defensive behavior has been noted, with a greater defensiveness for females toward a potential threat such as cat odor, as opposed to the actual presence of a cat [4]. When assessed by open field tests, emotionality is reduced in female rats in estrus [7]. Estrogen enhanced emotionality in female mice in elevated maze tests and induced a high level of grooming through induction of the progesterone receptor [18]. The MeA is among structures in which estrogen induces progesterone receptors [19]. Therefore, the MeA is well equipped, with its sexually dimorphism and the abundance of estrogen-induced progesterone receptor positive neurons, to co-ordinate estrogen-dependent, sex-specific behavior elicited by olfactory cues. Indeed, in response to predator odor, discrete Fos induction occurs in the posterodorsal part of the MeA [11]. The present MeA lesion encompassed the region in which Fos induction has been observed, suggesting a possible interface between olfactory input and emotional output in this structure.

Since the present study made lesions by radiofrequency rather than chemical substances that destroy neuronal soma but spare axons, the possibility of interruption of inputs to the basolateral and/or central nucleus of the amygdala cannot be excluded. However, histological examination revealed that lesions were sufficiently small and apart from the structures to disregard the influence of interrupting the afferents for emotional circuits comprised of basolateral/central amygdala.

In the paced mating test, the MeA lesion obviated the need for estrogen to decrease the latency to enter the male compartment and increased the likelihood that a female would stay with a male after sexual interactions regardless of the intensity of sexual stimuli. It is no wonder that the increase in the pacing was not detected in female rats carrying an MeA lesion, when they were tested following a combined treatment with estrogen and progesterone [20]. It is obvious that there is a close parallelism between the paced mating test and passive avoidance learning. Assuming that genital stimulation is aversive [6], entrance to the male compartment and subsequent sexual stimuli from the male would have an aversive value comparable to footshock. The loss of passive avoidance learning in the MeA lesioned females testifies to disturbances in emotional coping with aversive stimuli without affecting anxiety or emotionality as shown in the open field test.

The findings in the present study together demonstrate that two types of female behavior are affected by MeA lesion: the lesion made female rats indiscriminate toward sexually active and inactive males and reduced aversion to excessive sexual stimuli from the males. It should also be noted that the lesioned females do not respond to estrogen either in partner preference or pacing. It is to be seen which of the two types of estrogen receptor in the $\mathrm{MeA}, \alpha$ or $\beta$, is involved in the regulation of these behaviors.

This work was supported in part by Grants-in-Aid for Scientific Research (No. 12670068) from the Japan 
Society for the Promotion of Science.

\section{REFERENCES}

1. Akesson TR, Ulibarri C and Truitt S: Divergent axon collaterals originate in the estrogen receptive ventromedial nucleus of hypothalamus in the rat. J Neurobiol 25: 406-414, 1994

2. Bennett AL, Greco B, Blasberg ME, and Blaustein JD: Response to male odours in progestin receptorand oestrogen receptor-containing cells in female rat brain. J Neuroendocrinol 14: 442-449, 2002

3. Bermant G: Response latencies of female rats during sexual intercourse. Science 133: 1771-177, 1961

4. Blanchard DC, Shepherd JK, De Padua CA, and Blanchard RJ: Sex effects in defensive behavior: baseline differences and drug interactions. Neurosci Biobehav Rev 15: 461-468, 1991

5. Blanchard RJ, Nikulina JN, Sakai RR, McKittrick C, McEwen B, and Blanchard DC: Behavioral and endocrine change following chronic predatory stress. Physiol Behav 63: 561-569, 1998

6. Broekman M, de Bruin M, Smeenk J, Slob AK, and van der Schoot P.: Partner preference behavior of estrous female rats affected by castration of tethered male incentives. Horm Behav 22: 324-337, 1988

7. Burke AW and Broadhurst PL: Behavioural correlates of the oestrous cycle in the rat. Nature 209: 223-224, 1966

8. Cooke BM, Tabibnia G, and Breedlove SM: A brain sexual dimorphism controlled by adult circulating androgens. Proc Natl Acad Sci USA 96: 7538-7540, 1999

9. Coopersmith C, Candurra C, and Erskine MS: Effects of paced mating and intromissive stimulation on feminine sexual behavior and estrus termination in the cycling rat. J Comp Psychol 110: 176-186, 1996

10. Davis M: The role of the amygdala in fear and anxiety. Annu Rev Neurosci 15: 353-375, 1992

11. Dielenberg RA, Hunt GE, and McGregor IS: "When a rat smells a cat": the distribution of Fos immunoreactivity in rat brain following exposure to a predatory odor. Neuroscience 104: 1085-1097, 2001

12. Dielenberg RA, Hunt GE, and McGregor IS: "When a rat smells a cat": the distribution of Fos immunoreactivity in rat brain following exposure to a predatory odor. Neuroscience 104: 1085-1097, 2001

13. Eliasson M and Meyerson BJ: Sexual preference in female rats during estrous cycle, pregnancy and lactation. Physiol Behav 14: 705-710, 1975

14. Emery DE and Moss RL: p-Chlorophenylalanine alters pacing of copulation in female rats. Pharmacol Biochem Behav 20: 337-341, 1984

15. Erskine MS: Solicitation behavior in the estrous female rat: a review. Horm Behav 23: 473-502, 1989

16. Erskine MS and Hanrahan SB: Effects of paced mating on c-fos gene expression in the female rat brain. J Neuroendocrinol 9: 903-912, 1997

17. Ferguson JN, Aldag JM, Insel TR, and Young LJ: Oxytocin in the medial amygdala is essential for social recognition in the mouse. J Neurosci 21: 8278-8285, 2001

18. Galeeva AY, Tuohimaa P, and Shalyapina VG: The role of sex steroids in forming anxiety states in female mice. Neurosci Behav Physiol 33: 415-420, 2003

19. Greco B, Allegretto EA, Tetel MJ, and Blaustein JD: Coexpression of ER beta with ER alpha and progestin receptor proteins in the female rat forebrain: effects of estradiol treatment. Endocrinology 142: 5172-5181, 2001

20. Guarraci FA and Clark AS: Paced mating behavior in the female rat following ibotenic acid lesions of the medial amygdala. Soc Neurosci Abst . 2002. 
21. Guarraci FA, Megroz AB, and Clark AS: Paced mating behavior in the female rat following lesions of three regions responsive to vaginocervical stimulation. Brain Res 999: 40-52, 2004

22. Ichikawa M: Synaptic reorganization in the medial amygdaloid nucleus after lesion of the accessory olfactory bulb of adult rat. I. Quantitative and electron microscopic study of the recovery of synaptic density. Brain Res 420: 243-252, 1987

23. Jellestad FK, Markowska A, Bakke HK, and Walther B: Behavioral effects after ibotenic acid, 6-OHDA and electrolytic lesions in the central amygdala nucleus of the rat. Physiol Behav 37: 855-862, 1986

24. Kato A and Sakuma Y: Neuronal activity in female rat preoptic area associated with sexually motivated behavior. Brain Res 862: 90-102, 2000

25. Kelliher KR, Liu YC, Baum MJ, and Sachs BD: Neuronal Fos activation in olfactory bulb and forebrain of male rats having erections in the presence of inaccessible estrous females. Neuroscience 92: 1025-1033, 1999

26. Kondo Y and Sachs BD: Disparate effects of small medial amygdala lesions on noncontact erection, copulation, and partner preference. Physiol Behav 76: 443-447, 2002

27. Kondo Y, Sachs BD, and Sakuma Y: Importance of the medial amygdala in rat penile erection evoked by remote stimuli from estrous females. Behav Brain Res 88: 153-160, 1997

28. LeDoux J: Fear and the brain: where have we been, and where are we going? Biol Psychiatry 44: 1229-1238, 1998

29. LeDoux JE, Iwata J, Cicchetti P, and Reis DJ: Different projections of the central amygdaloid nucleus mediate autonomic and behavioral correlates of conditioned fear. J Neurosci 8: 2517-2529, 1988

30. Maisonnette SS, Kawasaki MC, Coimbra NC, and Brandao ML: Effects of lesions of amygdaloid nuclei and substantia nigra on aversive responses induced by electrical stimulation of the inferior colliculus. Brain Res Bull 40: 93-98, 1996

31. Maren S: Overtraining does not mitigate contextual fear conditioning deficits produced by neurotoxic lesions of the basolateral amygdala. J Neurosci 18: 3088-3097, 1998

32. Maren S: Long-term potentiation in the amygdala: a mechanism for emotional learning and memory. Trends Neurosci 22: 561-567, 1999

33. Maren S, Aharonov G, and Fanselow MS: Retrograde abolition of conditional fear after excitotoxic lesions in the basolateral amygdala of rats: absence of a temporal gradient. Behav Neurosci 110: 718-726, 1996

34. Maren S, Aharonov G, Stote DL, and Fanselow MS: N-methyl-D-aspartate receptors in the basolateral amygdala are required for both acquisition and expression of conditional fear in rats. Behav Neurosci 110: $1365-1374,1996$

35. Newman SW: The medial extended amygdala in male reproductive behavior. A node in the mammalian social behavior network. Ann N Y Acad Sci 877: 242-257, 1999

36. Oakes ME and Coover GD: Effects of small amygdala lesions on fear, but not aggression, in the rat. Physiol Behav 61: 45-55, 1997

37. Osterlund M, Kuiper GG, Gustafsson JA, and Hurd YL: Differential distribution and regulation of estrogen receptor-alpha and -beta mRNA within the female rat brain. Brain Res Mol Brain Res 54: 175-180, 1998

38. Paredes RG and Vazquez B: What do female rats like about sex? Paced mating. Behav Brain Res 105: $117-127,1999$

39. Paxinos $\mathrm{G}$ and Watson $\mathrm{C}$ : The rat brain in stereotaxic coordinates: compact third edition. Academic Press, San Diego, 1997

40. Sachs BD: Erection evoked in male rats by airborne scent from estrous females. Physiol Behav 62: 
921-924, 1997

41. Tashiro S, Kondo Y, and Sakuma Y: Temporal coincidence between the excitation of ventromedial hypothalamic efferents and the induction of lordosis reflex in ovariectomized estrogen-primed rats. Endocr J 45: 519-528, 1998

42. Vega MJ and Larsson K: Role of androgen, estrogen and sexual experience on the female rat's partner preference. Physiol Behav 50: 139-142, 1991

43. Xiao K, Kondo Y, and Sakuma Y: Sex-specific effects of gonadal steroids on conspecific odor preference in the rat. Horm Behav 46: 356-361, 2004

44. Xiao K, Kondo Y, and Sakuma Y: Differential regulation of female rat olfactory preference and copulatory pacing by the lateral septum and medial preoptic area. Neuroendocrinology 81:56-62, 2005

45. Yang LY and Clemens LG: MPOA lesions affect female pacing of copulation in rats. Behav Neurosci 114: 1191-1202, 2000

46. Yoshida M, Suga S, and Sakuma Y: Estrogen reduces the excitability of the female rat medial amygdala afferents from the medial preoptic area but not those from the lateral septum. Exp Brain Res 101: 1-7, 1994

47. Zipse LR, Brandling-Bennett EM, and Clark AS: Paced mating behavior in the naturally cycling and the hormone-treated female rat. Physiol Behav 70: 205-209, 2000

Table 1. Summary of results of open field test in sham and MeA-lesioned females. Ambulation was scored by number of lines crossed.

\begin{tabular}{lcc}
\hline \multicolumn{1}{c}{ Parameters } & Sham & MeAx \\
\hline Ambulation (outside) & $21.0 \pm 2.8$ & $24.6 \pm 1.6$ \\
Ambulation (center) & $39.8 \pm 2.9$ & $30.4 \pm 4.6$ \\
Number of rearings & $18.5 \pm 1.5$ & $13.7 \pm 2.1$ \\
Number of feces & $0.5 \pm 0.5$ & $0.3 \pm 0.2$ \\
\hline
\end{tabular}

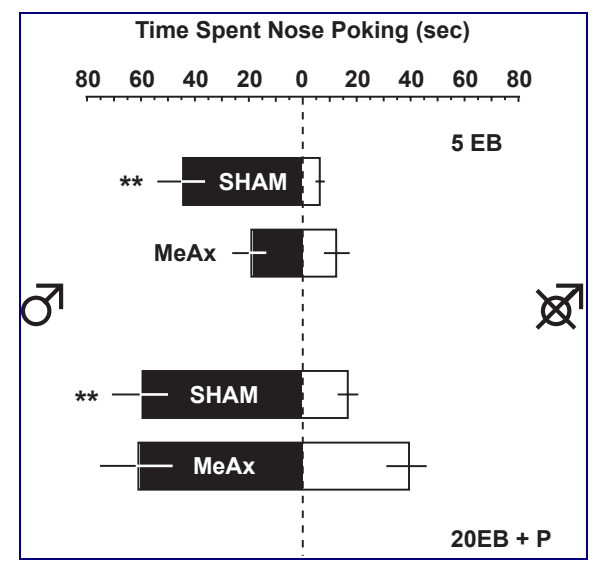

Fig. 1. Mean nose-poking time toward sexually active males (left side) and castrated males (right side) in partner preference test after injection of $5 \mu \mathrm{g}$ EB alone (5EB) or $20 \mu \mathrm{g}$ EB plus $500 \mu \mathrm{g} \mathrm{P}(20 \mathrm{~EB}+\mathrm{P})$. Horizontal bars in each column indicate SEM. ${ }^{* *} p<0.01$ for difference between sexually active and inactive males. 
This version is to be replaced by the final version after page-setting and proofing.

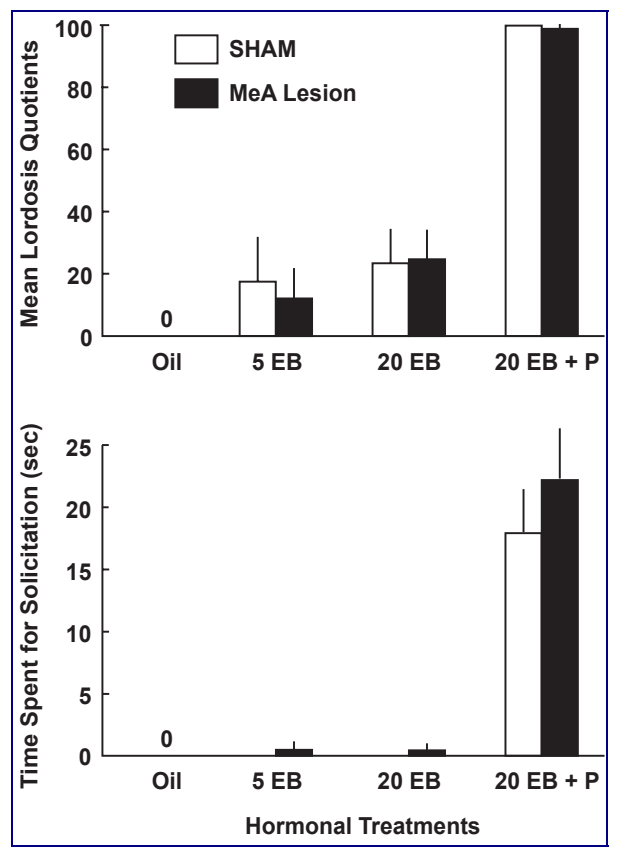

Fig. 2. Mean lordosis quotients (LQ, upper panel) and time spent in soliciting behavior (ear-wiggling and hopping/darting, lower panel) in paced mating test. Tests were carried out after 4 hormone treatment conditions (see abbreviations described in the legend to Fig. 1). Vertical bars in each column indicate SEM.

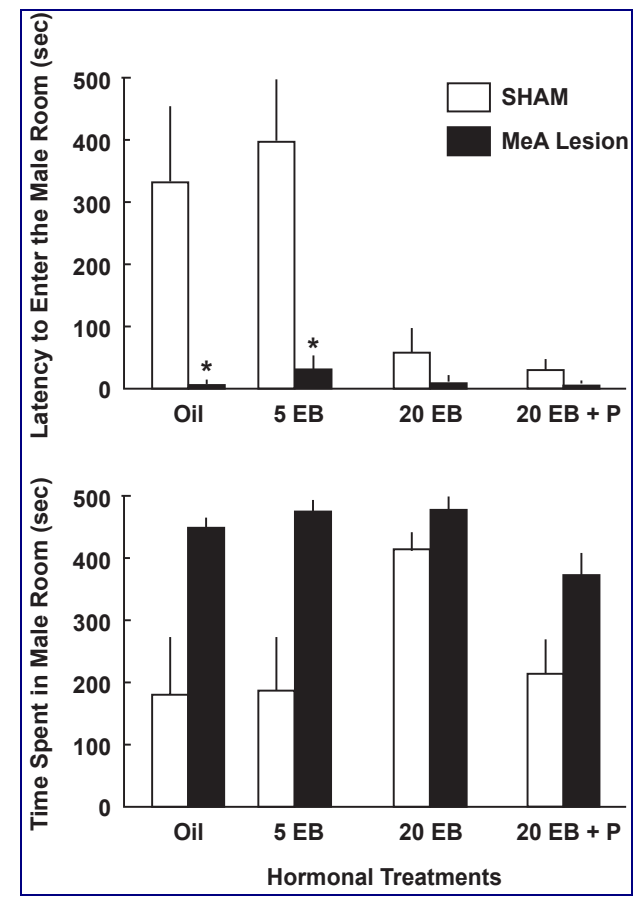

Fig. 3. Mean latency of entering (upper panel) and time spent (lower panel) in the male compartment in paced mating test. Tests were carried out after 4 hormone treatment conditions (see abbreviations described in the legend to Fig. 1). Vertical bars in each column indicate SEM. $* p<0.05$. 
This version is to be replaced by the final version after page-setting and proofing.

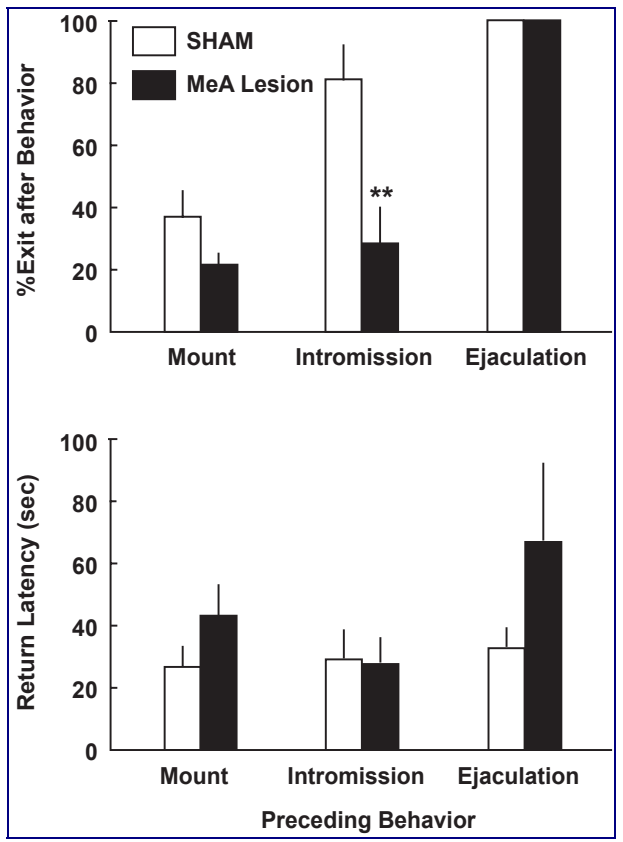

Fig. 4. Percent exits (upper panel) and return latency (lower panel) after each mount, intromission or ejaculation in the paced mating test in which females were injected with $20 \mu \mathrm{g}$ EB plus $500 \mu \mathrm{g} P$ $(20 E B+P)$. Vertical bars in each column indicate SEM. $* * p<0.01$.

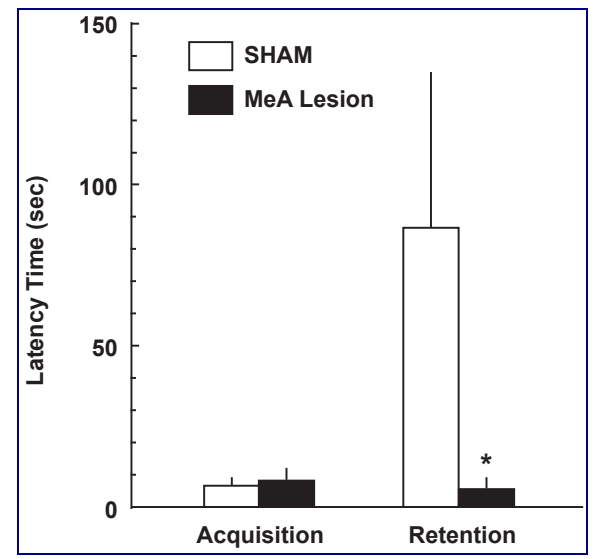

Fig. 5. Mean latency time to enter the black room before (acquisition) and after (retention) electrical grid-floor shock in passive avoidance test. Vertical bars in each column indicate SEM. ${ }^{*} p<0.05, U$-test.

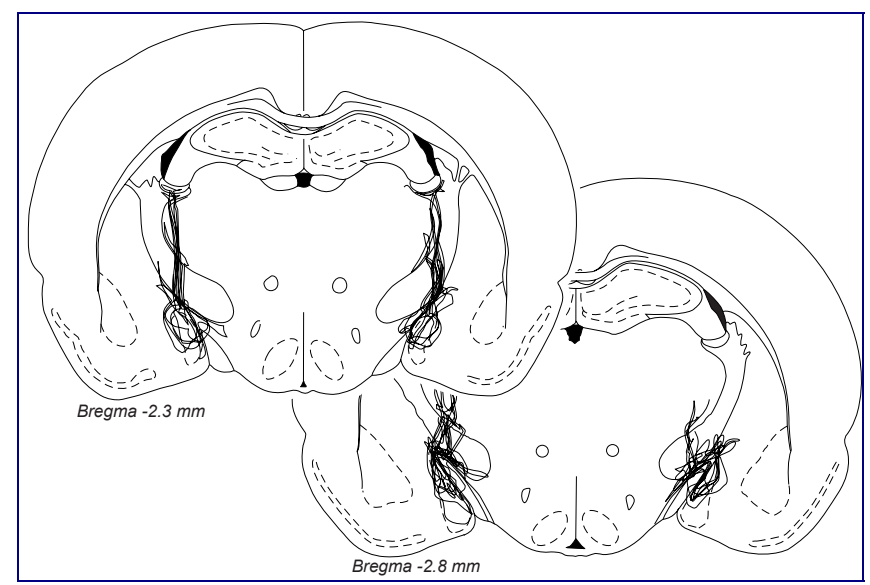


Fig. 6. Schematic drawing superimposed on all MeA lesions in the representative sections [39]. Values indicate AP distance from the bregma. 